\title{
Design and Realization of Jingdezhen Tourism E-commerce Website
}

\author{
Hu Jingfang, a , Li Busheng ${ }^{2, b}$ \\ ${ }^{1}$ School of Information Engineering, Jingdezhen Ceramic Institute, \\ Jingdezhen, 333403, China \\ ${ }^{2}$ School of Information Engineering, Jingdezhen Ceramic Institute, \\ Jingdezhen, 333403, China \\ aemail: jdzhjf@163.com, bemail:abulbs@163.com
}

\begin{abstract}
With the development of electronic commerce mode, the use of e-commerce of tourism has become a hot spot, this paper studies and analyzes the Jingdezhen tourism environment and tourism electronic commerce present situation, proposed the Jingdezhen tourism electronic commerce operation mode, key technology and further study the tourism website construction, finally realizes the construction of Jingdezhen tour business website.

Keywords: Electronic commerce; Operation mode; Jingdezhen tourism; Website

\section{Study on operation mode of Jingdezhen e-commerce of Tourism}

Jingdezhen is between the famous tourist scenic zone Lu shan, Huangshan and Sanqingshan, location advantages. From the tourism resources, Jingdezhen is rich in cultural relics and scenic spots, and the profound ceramic culture connotation. The natural scenery, famous historical, ceramic culture and folk customs constitute the Jingdezhen superior tourist resources, ceramic cultural resources are the advantages of Jingdezhen resources.

However, the development of tourism industry in Jingdezhen is not commensurate with its tourism resources, over the years the development level of Jingdezhen tourism industry in the country still lags behind. The huge contrast between the rich humanities, natural ecological of Jingdezhen and its tourism industry actual development situation, fully demonstrated the rich tourism resources has not been fully effective development and utilization. The basic reason is that the strategy and orientation of tourism development, did not seize the visitors psychological and embody the characteristics of Jingdezhen tourism resources.
\end{abstract}




\section{The analysis of the system function}

The biggest characteristic of tourism e-commerce website is intuitive, practical and open, it is different with the general tourism website lies in the realization of e-commerce. According to the actual need of tourism e-commerce website, which makes user interaction becomes smooth and continuous, to improve the availability and efficiency of the system. In order to present Jingdezhen tourism characteristics and attractions better, Jingdezhen tourism e- commerce website function with the settings shown below:

(1) User login and registration module: In the design of the system, this module is essential, is also relatively simple, it directly through the Jingdezhen tourism e- commerce platform. The new user registration need to fill in the user name, password, address, email, telephone numbers can complete the relevant registration. To provide the guarantee for the following operations. If the user is already a registered user, you can directly login link for subsequent operations, to provide a link for the new user registration service.

(2) A survey of Jingdezhen: Jingdezhen is rich in tourism resources, not only enjoy the "world porcelain" known, but also has favorable geographical conditions and natural scenery. The Castle Peak, green water, ceramic culture, revolutionary historical sites has profound cultural heritage and the local characteristics,occupies an important position in China. So we use the CSS to control the structure of the page style, writing folding menu, fast transmission of pictures, let the user feel the various styles of photo switching effect.

(3)Tourism news: The module is the main dynamic information in a timely manner to show attractions, allow the user to open the site you can see the information with new attractions, let users can feel the charm of scenic spots.

(4) Travel routes: in this module includes the territory of Jingdezhen and the surrounding many tourist routes. Play AJAX asynchronous request advantages technology, enhance the interactive method of WEB page based on JavaScript in the process of specific implementation in WEB. We put the graphics knowledge to work by WEB development. First of all, we can use graphics algorithms to process the pictures. Secondly, using the JavaScript and AJAX can reduce user waiting time in image processing and image transmission such a huge amount of data operation events, which makes user interaction becomes smooth and continuous.

(5) Booking tickets and hotel reservation: This is a key part of the electronic commerce website difference between tourism and general tourism website. General travel websites simply to introduce attractions and line .Tourism e-commerce site can provide tickets for scenic spots, lines, hotel online booking.

(6) Specialty Mall: in order to give the user understands and buy local specialty goods in tourism.

(7) Personal information center: When the user opens a website, encounter problems, you can view some of the problems by the help center answers, so as 
to help the user to solve the problem. Below is a picture of the overall structure of the system design.

Below is the structure design of the system (fig.1):

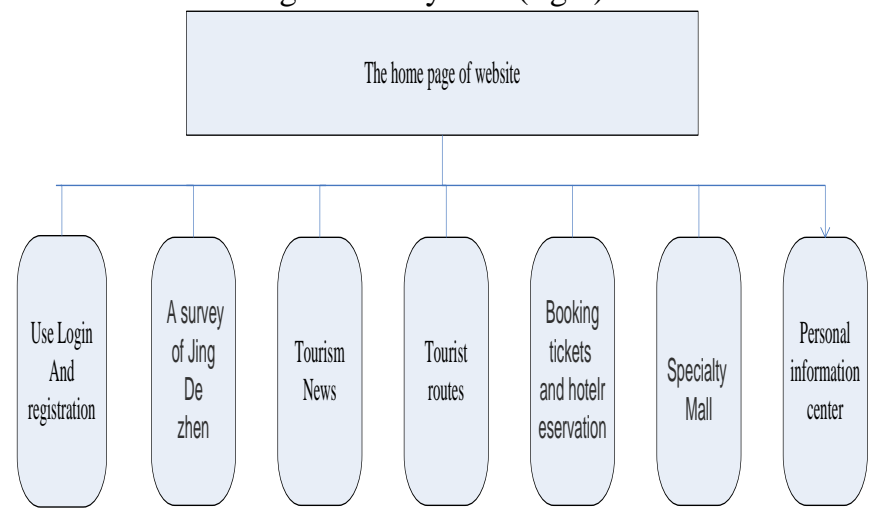

Fig.1. Website structure diagram

\section{Realization method of main technology}

The system uses the web development language is PHP5.5, database is MySql 5.26, the operating system is Windows $\mathrm{xp} / \mathrm{Win} 7$. The main core technology is mainly: PHP technology, database technology, and Web Technology (html+ css, javascript + jquery + ajax).

The first is the basic operation of MySql, the mainly is the design of the database, and the E-R model of database, the following is the simple E-R model of the system(Fig.2).

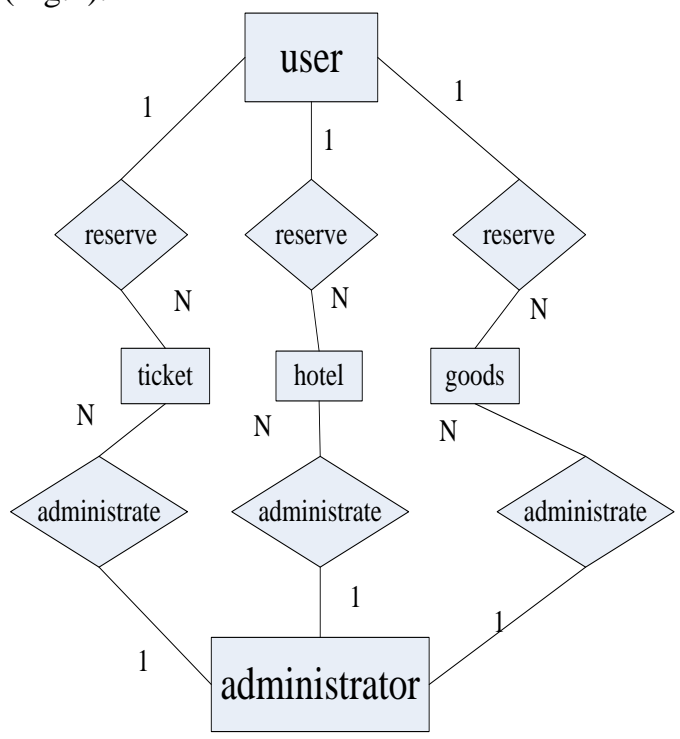

Fig.2. The simple E-R model of the system 
PHP operation of MySQL is divided into 5 steps: (here only to illustrate the MySql )

The first step: to connect (here my database username is root, password is 23456)

\$conn=mysql_connect("localhost","root","123456");

The second step: setting

mysql_query("set names utf8");

The third step: Perform

\$sql="select * from tb_news";

\$result=mysql_query(\$sql,\$conn) or die(mysql_error());

The fourth step: the result

Here need to see the results returned is the result set, or a simple digital, there are a lot of methods of treatment results, mainly through the index array or associative array, the following simple processing is obtained on the basis of the result of the previous step to deal with:

\$rowCount=mysql_num_rows(\$result);

The fifth step: close the connection

mysql_free_result(\$result);

Mysql_close(\$conn);

The following will explain the parameters transfer technology .There are several methods of parameter transfer, here we will only explain session and cookie. Session and cookie are used as variables in the PHP, such as:

\$_SESSION ['name']="zhangsan”,\$_COOKIE['name']="zhangsan”;

Webj Technology: the first is html+css, this is the layout of the page. Then is JavaScript, this is a dynamic interactive of pages. And last is the jquery and Ajax, AJAX is the combination of JavaScript and background data.

Baidu map interface technology: here use the open platform of Baidu developer, which calls the interface can realize the online map:

Use a JavaScript file

<script src="http://api.map.baidu.com/api?v=1.4"

type="text/javascript" $></$ script $>$

$<$ script type="text/javascript">

var map = new BMap.Map("container"); // Create map examples

map.setDefaultCursor("crosshair");//Set the default mouse pointer style of map

map.enableScrollWheelZoom();// Enable wheel zoom, disabled by default.

var point = new BMap.Point(117.17842, 29.2688); // Create point coordinate map.centerAndZoom(point, 15); // Initialize the map, sets the center point coordinate map.addControl(new BMap.NavigationControl()); // Add the zoom control of ground map.addControl(new BMap.ScaleControl()); // Add scale control map.addControl(new BMap.OverviewMapControl()); // A thumbnail map control

map.addControl(new BMap.MapTypeControl()); // Map type control map.setCurrentCity("JingDezhen"); 


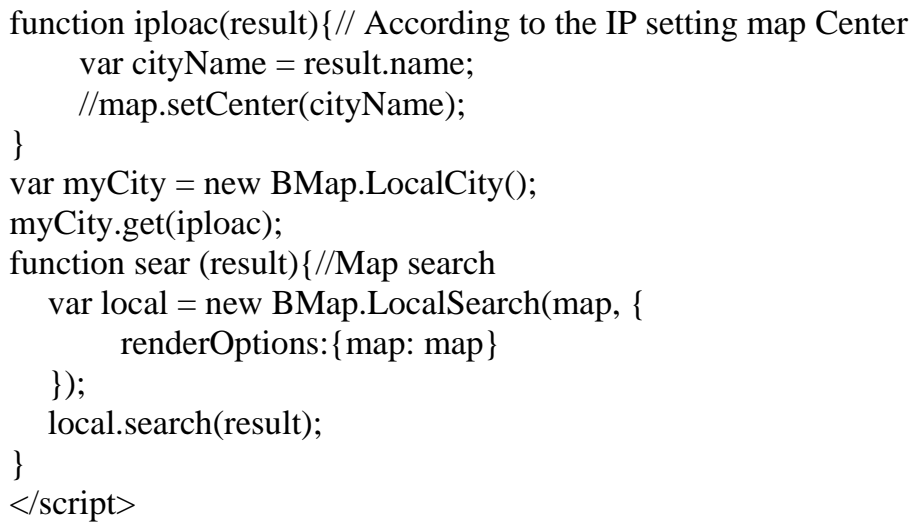

\section{Conclusion}

Through the analysis and design, we completed the tourism e-commerce system based on B/S mode. The system contains news system, user registered and login system, background management system, tickets and hotel reservation system, specialty store, the help center module.

The research of this topic is the research based on the industry perspective on Jingdezhen tourism industry to actively develop e-commerce problems. By the time, funds and technical conditions limit, there are many practical problems to be further studied. In the construction of Jingdezhen tourism website, we should improve the service functions of tourism website. We also should establish online travel consultation and service system in the website, make those needs related consulting customers can get help. Finally, in the actual operation, we also need to improve the payment function.

\section{References}

[1] Chenhao. PHP program design [M] Beijing: Electronics industry Press, 2005

[2] ShaoYu. PHP and MYSQL WEB development [M]. Beijing: Mechanical Industry Press, 2005

[3] DAVID LANE .PHP \& MYSQL WEB Database application development Guides [M].Nanjing: Southeast University Press, 2006

[4] MIHAI BUCICA. AJAX and PHP WEB development [M].Beijing: People's Posts and Telecommunications Press, 2007

[5] PETER MOULDING. PHP technology insider [M].Beijing: China Waterpower Press, 2003 
[6] NARAMORE. PHP5、APACHE、MYSQL web development [M].Beijing: Electronics industry Press, 2005

[7] JiGenlin CuiHaiyuan. Web program design [M]. Beijing: Electronics industry Press, 2008.4

[8] Simon Collision. CSS foundation tutorial [M]. Beijing: People's Posts and Telecommunications Press, 2007. 